\title{
Technologiegestütztes Lehren und Lernen in der Medizin
}

\author{
Martin Haag ${ }^{1}$ \\ 1 GECKO-Institut für Medizin, \\ Informatik und Ökonomie, \\ Fakultät für Informatik, \\ Hochschule Heilbronn, \\ Heilbronn, Deutschland
}

\section{Editorial}

Die Informationstechnologie hat sich in den zurückliegenden Jahren rasant fortentwickelt und hat zu starken Veränderungen unseres privaten und beruflichen Alltags geführt. Information und Wissen sind heute wesentlich komfortabler und schneller verfügbar, als dies noch vor einigen Jahren der Fall war. Technologische Treiber der Entwicklung waren und sind hierbei das World Wide Web und der weltweit starke Ausbau der Internets. Darüber hinaus ist die rasante Verbreitung von mobilen Endgeräten wie Smartphones und die zunehmende Verfügbarkeit schneller Funknetze zu nennen, die auch unterwegs einen breitbandigen Zugang zum Internet ermöglichen. Innovative neue Technologien ermöglichen uns, Lehre an Universitäten und Fachhochschulen interessanter und idealerweise lernwirksamer zu gestalten sowie ganz neue Formen des Lehrens und Lernens zu entwickeln, nicht nur für die Präsenzlehre, sondern auch für das lebenslange Lernen.

Aufgrund der beschriebenen rasanten technologischen Entwicklung wurde die seit mehr als 20 Jahren bestehende gmds-Arbeitsgruppe „Computerunterstützte Lehr- und Lernsysteme in der Medizin (CBT)“ 2012 in Arbeitsgruppe „Technologiegestütztes Lehren und Lernen in der Medizin (TeLL)“ umbenannt. Damit soll dokumentiert werden, dass sich die Arbeitsgruppe nicht nur mit der Erstellung von Lernsystemen für klassische PCs beschäftigt, sondern auch neue technologische Trends aufgreift und erforscht, inwieweit sich diese für die Lehre sinnvoll nutzen lassen. An den Programmen der CBT-Workshops der Arbeitsgruppe lässt sich die dargestellte Entwicklung sehr schön verfolgen. In 2011 fand der 15. Workshop vom 7. bis zum 8. April 2011 in Berlin unter dem Motto „Lebenslanges Lernen mit innovativen Technologien: Anspruch und Wirklichkeit aktueller E-Learning-Szenarien in den Gesundheitsberufen“ statt. In bereits bewährter Weise wurde dieser gemeinsam mit dem Ausschuss „Neue Medien in der Medizinischen Ausbildung“ der Gesellschaft für Medizinische Ausbildung ausgerichtet. Aus dem Kreise der Referentinnen und Referenten konnten erfreulicherweise einige Personen gefunden werden, die einen Beitrag für diese Zeitschrift erstellt haben. Alle eingereichten Beiträge

wurden dem üblichen Peer-Review-Verfahren der GMS Medizinische Informatik, Biometrie und Epidemiologie (MIBE) unterzogen. Insgesamt haben drei Arbeiten den Review-Prozess erfolgreich durchlaufen. Die Originalarbeit von Tolks und Fischer (http://www.egms.de/en/journals/ mibe/2013-9/mibe000131.shtml) beschäftigt sich mit Serious Games for Health und stellt dar, welche Serious Games for Health geeignet erscheinen um diese in medizinische Curricula einzubinden. Die Originalarbeit von Behrends et al. beschreibt Konzepte eines Online-Studiengangs für Hebammen an einer Präsenzuniversität (http:// www.egms.de/en/journals/mibe/2013-9/ mibe000130.shtml). Die Erfahrungen aus diesem Beitrag sollten sich auf weitere berufsbegleitende Studiengänge für andere Berufsgruppen im Gesundheitswesen übertragen lassen. Gerade für Master-Studiengänge erscheinen berufsbegleitende Studienangebote immer wichtiger zu werden, weil nach Beobachtungen an verschiedenen Hochschulen ein relativ hoher Anteil der Master-Studierenden neben dem Studium arbeitet, um den Lebensunterhalt bestreiten zu können.

Die dritte Originalarbeit von Sturm und Igel beschreibt, wie mittels einer App für Smartphones Studierende aller Fachrichtungen komfortabel auf ein Lernmanagementsystem zugreifen können (http://www.egms.de/en/ journals/mibe/2013-9/mibe000129.shtml). Gerade für Medizinstudierende ergibt sich hierdurch ein großer Nutzen, weil sich durch die mittlerweile sehr verbreitete Blockung von Lehrveranstaltungen und Modulen der Stundenplan häufig ändert. Durch die beschriebene App kann von unterwegs jederzeit auf alle relevanten Informationen wie Veranstaltungsort und auf die kursbezogenen Lerninhalte zugegriffen werden. Apps für mobile Geräte bieten noch viel Potential für innovative neue Qualifizierungsformen im lebenslangen Lernprozess für alle Berufsgruppen im Gesundheitswesen. Die Entwicklung in diesem Bereich steckt freilich noch in den Kinderschuhen.

Besonderer Dank gebührt dem Organisationsteam des 15. CBT-Workshops in Berlin um Kai Sostmann. Er hat mit seinem Team den Workshop hervorragend organisiert und ein hochattraktives Programm zusammengestellt, aus dem heraus die vorliegenden Originalarbeiten entstanden sind. Der Dank gilt auch allen Gutachtern, die 
an der Begutachtung der eingereichten Beiträge mitgewirkt haben.

\section{Korrespondenzadresse:}

Martin Haag

GECKO-Institut für Medizin, Informatik und Ökonomie,

Fakultät für Informatik, Hochschule Heilbronn,

Max-Planck-Str. 39, 74081 Heilbronn, Deutschland, Tel.:

$+49 /(0) 7131 / 504497$

martin.haag@hs-heilbronn.de
Bitte zitieren als

Haag M. Technologiegestütztes Lehren und Lernen in der Medizin. GMS Med Inform Biom Epidemiol. 2013:9(1):Doc04.

DOI: 10.3205/mibe000132, URN: urn:nbn:de:0183-mibe0001325

Artikel online frei zugänglich unter

http://www.egms.de/en/journals/mibe/2013-9/mibe000132.shtml

Veröffentlicht: 31.01 .2013

\section{Copyright}

(c)2013 Haag. Dieser Artikel ist ein Open Access-Artikel und steht unter den Creative Commons Lizenzbedingungen

(http://creativecommons.org/licenses/by-nc-nd/3.0/deed.de). Er darf vervielfältigt, verbreitet und öffentlich zugänglich gemacht werden, vorausgesetzt dass Autor und Quelle genannt werden. 